Article

\title{
Soft Neutrosophic Modules
}

\author{
Mikail Bal and Necati Olgun *(D) \\ Department of Mathematics, Gaziantep University, Gaziantep 27310, Turkey; mikailbal46@hotmail.com \\ * Correspondence: olgun@gantep.edu.tr
}

Received: 22 October 2018; Accepted: 8 December 2018; Published: 12 December 2018

\begin{abstract}
In this study, for the first time, we study some basic definitions of soft neutrosophic modules in algebra being generalized and its several related properties, structural characteristics are investigated with suitable examples. In this paper, we utilized neutrosophic soft sets and neutrosophic modules. As a result, we defined soft neutrosophic modules. After weak soft neutrosophic modules and strong soft neutrosophic modules are described and illustrated by examples. Finally soft neutrosophic module homomorphism is defined and soft neutrosophic module isomorphism is explained.
\end{abstract}

Keywords: strong soft neutrosophic module; weak soft neutrosophic module; soft neutrosophic submodule; soft neutrosophic ring

\section{Introduction}

In 1980, Florentin Smarandanche characterized the notion of neutrosophy as a new branch of philosophy. "It is a branch of philosophy which studies the nature, scope and origin of neutralities, as well as their interactions with different ideational spectra". It is the base of neutrosophic logic that is an extension fuzzy logic in that indeterminacy is inclusive. He introduced the concept of (T-truth, I-indeterminacy, and F-falsity) memberships. Neutrosophic set is a power general formal structure which generalizes the concept of the intuitionistic fuzzy set, interval valued fuzzy set, fuzzy set, classic set etc.

Struggle with uncertainties is a big problem in many areas like medical science, environment, economics, social sciences, etc. These kinds of problems cannot be overcome by classical methods, because there are inherent difficulties in the classical methods. The reason for these difficulties may be due to the inadequacy of the theories of parameterization tools. For Dealing with these kinds of difficulties, Molodtsov suggested a new mathematical tool for dealing with uncertainties. This is called soft set theory [1].

The algebraic structure of set theories dealing with uncertainties has also been studied by some authors. Smarandanche and others, studied neutrosophic algebraic structures [2-4] by using the idea of neutrosophic theory and this was the first neutrosofication of algebraic structures. Some authors [2-21] have studied neutrosophic algebraic structures and nowadays, researchers are still interested in this subject. The importance of this neutrosophic structures continues to increase. Recently, researchers have done a number of soft neutrosophic structures formed by combining soft structures and neutrosophic structures $[18,20,21]$.

Therefore, we decided to investigate the effects of this subject on modules of algebraic structures. Modules are basic structures in algebraic structures and are generalization of the vector spaces. This topic has not been studied yet.

In this paper, for the first time we introduce 'Soft Neutrosophic Module', 'Weak Soft-N Module', and Strong Soft- $N$ Module'. Finally, we described the soft- $N$ module homomorphism and the soft- $N$ module isomorphism in module theory, using neutrosophic soft sets. This is new topic in the module 
theory and neutrosophic logic. The rest of the paper is organized as follows. After the literature review in Section 1 and Preliminaries in Section 2, we introduce soft neutrosophic module, weak soft- $N$ module and strong soft- $N$ module and also examine the relations between weak soft- $N$ module and strong soft- $N$ module in Section 3. Finally, conclusions are given in Section 4.

\section{Preliminaries}

In this chapter, we remember some basic definition for soft sets and neutrosophic modules. These are related to fuzzy sets, soft sets, neutrosophic sets which are found in the literature $[1,2,5,22-26]$.

\subsection{Soft Sets Theory}

Let $U$ be an initial universe set and $E$ be a set of parameters. The power set of $U$ is denoted by $P(U)$ and $A \subset E$. Molodtsov defined the notation of a set in the following way:

Definition 1. A pair $(F, A)$ is called a soft set over $U$, where $F$ is a mapping given by:

$$
F: A \rightarrow P(U)[1]
$$

Definition 2. For two soft sets $(F, A)$ and $(G, B)$ over a general universe $U$, we say that $(F, A)$ is a soft subset of $(G, B)$ if:

(i) $A \subset B$, and

(ii) $F(x)$ and $G(x)$ are identical approximations $\forall \mathrm{x} \in A$. We write $(F, A) \subset(G, B)$.

$(F, A)$ is said to be a soft super set of $(G, B)$ if $(G, B)$ is a soft subset of $(F, A)$. We denote it by $(F, A) \supset(G, B)$ (see [1]).

Definition 3. Two soft sets $(F, A)$ and $(G, B)$ on a common universe $U$ are said to be soft equal if $(F, A)$ is a soft subset of $(G, B)$ and $(G, B)$ is a soft subset of $(F, A)$ (see [1]).

Definition 4. Union of two soft sets of $(F, A)$ and $(G, B)$ over the common universe $U$ is the soft set $(H, C)$, where $C=A \cup B$, and $\forall \mathrm{x} \in C$,

$$
\begin{aligned}
& H(e) \quad=F(x), \quad \text { if } x \in A-B \text {, } \\
& =(G), \quad \text { if } x \in B-A \text {, } \\
& =F(x) \cup G(x) \text {, if } x \in A \cap B \text {. }
\end{aligned}
$$

We write:

$$
(F, A) \cup(G, B)=(H, C)(\text { see }[1]) .
$$

Definition 5. Intersection of two soft sets $(F, A)$ and $(G, B)$ over a common universe $U$ is the soft set $(H, C)$, where $C=A \cap B$, and $\forall \mathrm{x} \in C, H(x)=F(x)$ or $G(x)$, (as both are same set). We write:

$$
(F, A) \cap(G, B)=(H, C)(\text { see }[1]) .
$$

\subsection{Neutrosophic Theory}

Definition 6. Let $\left(G,{ }^{*}\right)$ be any group and $\langle G \cup I\rangle=\{x+y I: x, y \in G\}$ be a set. Then $(<G \cup I\rangle$ $, *)=N(G)$ is called a neutrosophic group generated by I and $G$ under the binary operation $*$. I is called the neutrosophic element with the property $I^{2}=I$. For an integer $n, n+1$, and $n I$ are neutrosophic elements and $0 . I$ $=0 . I^{-1}$, the inverse of I is not defined and hence does not exist (see [2]).

Theorem 1. Let $(<G \cup I\rangle, *)=N(G)$ be the neutrosophic group. Then:

(i) $N(G)$ always contains a group.

(ii) $N(G)$ is not a group in general (see [2]). 
Definition 7. Let $<G \cup I>=N(G)$ be a neutrosophic group generated by I and $G$. A proper subset $P(G)$ is said to be a neutrosophic subgroup if $P(G)$ is a neutrosophic group (i.e., $P(G)$ should contain a subgroup) (see [5]).

Definition 8. Let $N(G)$ be any neutrosophic group. If $a b=$ ba for each $a, b \in N(G)$, then $\left(N(G),{ }^{*}\right)$ is called $a$ commutative neutrosophic group (see [5]).

Theorem 2. Let $N(P)$ and $N(M)$ be any two neutrosophic subgroups of a commutative neutrosophic group $N(G)$. Then:

(i) $\quad N(P) \cap N(M)$ is a neutrosophic subgroup of $N(G)$.

(ii) $N(P) . N(M)$ is a neutrosophic subgroup of $N(G)$,

(iii) $N(P) \cup N(M)$ is a neutrosophic subgroup of $N(G)$ if and only if $N(M) \subseteq N(P)$ or $N(P) \subseteq N(M)$ (see [5]).

Definition 9. Let $\left(R,+,{ }^{*}\right)$ be any ring. The set $\langle R \cup I>=\{x+y I: x, y \in R\}$ is neutrosophic ring generated by $I$ and $R$ under the operation of $R$ (see [5]).

Theorem 3. If $<R \cup I>$ a neutrosophic ring, then $<R \cup I>$ is a ring (see [5]).

Definition 10. Let $\langle R \cup I\rangle$ be a neutrosophic ring, We say that $\langle R \cup I\rangle$ is commutative neutrosophic ring if $a b=b a \forall a, b<R \cup I>($ see [5]).

\section{Soft Neutrosophic (Soft-N) Modules}

Definition 11. Let $((H, A),+,$.$) be any soft G$-module $(G-m o d)$ over a commutative soft ring $(G, A)$ and let $(H$, A) $(I)=<(H, A) \cup I>$ be a soft-N set generated by $(H, A)$ and $I$. The triple $((H, A)(I),+,$.$) is defined a weak$ soft-N G-mod over a soft ring $(G, A)$. In case of $(G, A)(I)$ is a soft-N G-mod over a soft-N ring $(G, A)(I)$, it is called a strong soft-N G-mod. The elements of $(H, A)(I)$ are called soft-N elements and the elements of $(G, A)(I)$ are described soft- $N$ scalars.

If $a_{1}=k+m I, a_{2}=n+p I \in H(I)$ where $k, m, n, p \in(H, A)$ and $q=u+v I \in(G, A)(I)$ where $u, v$ are scalars in $G$, we define:

$$
\begin{gathered}
a_{1}+a_{2}=(k+m I)+(n+p I)=(k+n)+(m+p) I \text { and } \\
q a_{1}=(u+v I) \cdot(k+m I)=u k+(u m+k v+v m) I .
\end{gathered}
$$

Example 1. Let $(G, A)$ be a commutative soft ring and $K$ be an ideal of $(G, A)$.

(i) $(G, A)(I)$ is a soft-N G-mod itself, because $(G, A)(I)$ is a soft-N abelian group. Hence the multiplication in G gives us a mapping:

$$
G \times(G, A)(I) \rightarrow(G, A)(I) .
$$

The soft-N ring axioms provide that this scalar multiplication turns G into a soft-N G-mod.

(ii) $(G, A)(I)$ is a weak soft-N G-mod on a soft ring $(Q, A)$. Moreover this is a strong soft-N G-mod on a soft- $N$ ring $(Q, A)(I)$.

(iii) If $K$ is closed under addition and multiplication by arbitrary elements of soft $(G, A)(I)$, then $K$ is an soft-N G-mod under the (+) and (.) of $G$,

(iv) $(G, A)_{n}(I)$ is a weak soft-N G-mod on a soft ring $(G, A)$. Moreover this is a strong soft-N G-mod over a $\operatorname{soft}-N \operatorname{ring}(G, A)(I)$.

(v) $(H, A)_{m \times n}(I)=\left\{\left[a_{i j}\right]: a_{i j} \in(Q, A)(I)\right\}$ is a weak soft-N G-mod on a soft ring $(Q, A)$. Moreover this is a strong soft-N G-mod on a soft-N ring $(Q, A)(I)$.

Theorem 4. Each strong soft-N G-mod is a weak soft-N G-mod.

Proof. Let $(H, A)(I)$ be a strong soft- $N$ module over a soft-N ring $(G, A)(I)$. We have, $(G, A) \subseteq(G, A)(I)$ is true for every soft $\operatorname{ring}(G, A)$. Therefore $(H, A)(I)$ is a weak soft-N G-mod. 
Theorem 5. Each weak (strong) soft-N G-mod is a soft G-mod.

Proof. Assume that $(H, A)(I)$ is a strong soft- $N$ module over a soft- $N$ ring $(G, A)(I)$. Clearly visible, $((H, A)(I),+,$.$) is an abelian soft group. Let a_{1}=k+\mathrm{mI}, a_{2}=n+p I \in(H, A)(I), q=x+y I, w=z+t I \in(G$, A) $(I)$ where $k, m, n, p \in(H, A)$ and $x, y, z, t \in G$. Then:

$$
\begin{gathered}
\text { (1) } q\left(a_{1}+a_{2}\right)=(x+y I)(k+m I+n+p I) \\
=x k+x n+[x m+x p+y k+y m+y n+y p] I \\
=(x+y I)(k+m I)+(x+y I)(n+p I) \\
=q a_{1}+q a_{2} . \\
(2)(q+w) a_{1}=(x+y I+z+t I)(k+m I) \\
=x k+z k+[x m+z m+y k+t k+y m+t m] I \\
=(x+y I)(k+m I)+(z+t I)(k+m I) \\
=q a_{1}+w a_{1} \\
\text { (3) }(q w) a_{1}=((x+y I)(z+t I))(k+m I) \\
=x z k+[x z m+x t k+y z k+y t k+x t m+y z m+y t m] I \\
=(x+y I)((z+t I)(k+m I)) \\
=q\left(w a_{1}\right)
\end{gathered}
$$

(4) For $1+1+0 I \in G(I)$, we have

$$
\begin{aligned}
1 a_{1}= & (1+1 I)(k+m I) \\
= & 1 k+1 m I \\
= & k+m I=a_{1} .
\end{aligned}
$$

Consequently, $(H, A)(I)$ is a soft G-mod.

Lemma 1. Assume that $(H, A)(I)$ is a strong soft-N G-mod over a soft-N ring $(G, A)(I)$ and let $a_{1}=k+m I, a_{2}$ $=n+p I, a_{3}=u+v I \in(H, A)(I), q=x+y I \in(G, A)(I)$. Then:

(i) $a_{1}+a_{3}=a_{2}+a_{3}$ implies $a_{1}=a_{2}$.

(ii) $q 0=0$.

(ii) $0 a_{1}=0$.

(iv) $(-q) a_{1}=q\left(-a_{1}\right)=-\left(q a_{1}\right)$.

Proof. (i) Let $a_{1}+a_{3}=a_{2}+a_{3}$. Then $(k+m I)+(u+v I)=(n+p I)+(u+v I)$. Clearly we obtain $(k+m I)=$ $(n+p I)$ from definition of the strong soft-N G-mod.

(i) $\quad q(0+0 I)=(x+y I)(0+0 I)=(x 0+\mathrm{x} 0+\mathrm{y} 0+y 0 I)=(0+0 I)=0$

(ii) $0 a_{1}=0(k+m I)=0$

(iii) $(-q) a_{1}=(-x-y I)(n+p I)=(-x n-x p I-y n I-y p I) q\left(-a_{1}\right)=(x+y I)(-n-p I)=(-x n-x p I-$ $y n I-y p I)-\left(q a_{1}\right)=-((x+y I)(n+p I))=(-x n-x p I-y n I-y p I)$.

Hence we obtain the equality $(-q) a_{1}=q\left(-a_{1}\right)=-\left(q a_{1}\right)$.

Definition 12. Let $(H, A)(I)$ be a strong soft-N G-mod on a soft-N ring $(G, A)(I)$ and $\varnothing \neq(N, A)(I) \subset(H$, A) (I). If $(N, A)(I)$ is itself a strong soft-N G-mod over $(G, A)(I)$, then it is called a strong soft-N submodule of $(M, A)(I)$. It is necessary that $(M, A)(I)$ must contains a proper soft subset which is a soft G-mod.

Definition 13. Let $(H, A)(I)$ be a weak soft-N G-mod over a soft ring $(G, A)$ and $\varnothing \neq(N, A)(I) \subset(H, A)(I)$. if $(N, A)(I)$ is itself a weak soft-N G-mod over $(G, A)$, then it is called a weak soft-N submodule of $(H, A)(I), I t$ 's necessary that $(N, A)(I)$ must contains a proper subset which is a soft G-mod.

Theorem 6. Let $(H, A)(I)$ be a strong soft-N G-mod on a soft-N ring $(G, A)(I)$ and $\varnothing \neq(N, A)(I) \subset(H, A)$ (I). Then $(N, A)(I)$ is a strong soft-N submodule of $(H, A)(I)$ if and only if the following conditions hold: 
(i) $a_{1}, a_{2} \in(N, A)(I)$, implies $a_{1}+a_{2} \in(N, A)(I)$.

(ii) $a_{1} \in(N, A)$ (I) and $q=x+y I \in(G, A)$ (I) for all $x, y \in G$ implies $q a_{1} \in(N, A)$ (I).

(iii) $(N, A)(I)$ must include a proper soft subset which is a soft G-mod.

Proof. $(\Rightarrow)$ This is clear, since $(N, A)(I)$ must contain the zero element 0 of $(H, A)(I)$, and $(N, A)(I)$ must be closed under the addition of $(H, A)(I)$ and under scalar multiplication by arbitrary elements of $(G, A)(I)$.

$(\Leftarrow)$ By the Subgroup Criterion, $(N, A)(I)$ is an additive subgroup of $(H, A)(I)$; also, by (ii), $(N, A)$ (I) is closed under scalar multiplication by arbitrary elements of $(G, A)(I)$. It is now clear that module properties are automatically inherited from $(H, A)(I)$.

If (i) and (ii) are combined in the above theorem, we can write as follows.

Corollary 1. Let $(H, A)(I)$ be a strong soft-N G-mod on a soft-N ring $(G, A)$ (I) and let $\varnothing \neq(N, A)(I) \subset(H$, A) (I). Then $(N, A)(I)$ is a strong soft-N submodule of $(M, A)(I)$ if and only if the following conditions hold:

(i) $a_{1}, a_{2} \in(N, A)(I)$ and every $q, w \in(G, A)(I)$, implies $q a_{1}+w a_{2} \in(N, A)(I)$

(ii) $\quad(N, A)(I)$ must include a proper soft subset which is a soft G-mod.

Example 2. Let $(H, A)$ (I) be a weak (strong) soft-N G-mod. Then $(H, A)(I)$ is a weak (strong) soft-N submodule of itself. It is called a trivial weak (strong) soft-N submodule.

Example 3. Let $(H, A)(I)=(G, A)(I)$ be a strong soft-N G-mod on a soft-N ring $(G, A)(I)$. The set $(N, A)(I)=$ $\left\{\left(a_{1}=x+y I, a_{2}=z+t I, 0=0+0 I\right) \in(H, A)(I): x, y, z, t \in(H, A)\right\}$ is a strong soft-N submodule of $(H, A)(I)$.

Example 4. Let $(H, A)(I)=(H, A)_{m_{n}^{*}}(I)=\left\{\left[x_{i j}\right]: x_{i j} \in(H, A)(I)\right\}$ be a strong soft-N G-mod on $(G, A)(I)$. The set of soft matrices $(N, A)(I)=(H, A)_{m^{*} n}(I)=\left\{\left[y_{i j}\right]: y_{i j} \in(G, A)(I)\right.$ and trace $\left.(H)=0\right\}$ is a strong soft-N submodule of $(G, A)(I)$.

Theorem 7. Assume that $(H, A)(I)$ is a strong soft-N G-mod on a soft-N ring $(G, A)(I)$ and $\left\{(N, A)_{n}(I)\right\}_{n \in \lambda}$ is a family of strong soft-N submodules of $(H, A)(I)$. Then $\cap(N, A)_{n}(I)$ is a strong soft-N submodule of $(H, A)(I)$.

Proof. Obviously, $0_{(H, A)} \cap(N, \mathrm{~A})_{\mathrm{n}}(I)$ for $\left.\forall n 0_{(M, A)}, \mathrm{A}\right)_{\mathrm{n}}\left(I\right.$ and hence $\cap(N, A)_{\mathrm{n}}(I) \neq \varnothing$. Let $x, y \cap(N$, $A)_{\mathrm{n}}(I)$ and let $a(G, A)(I)$ soft $N$-ring. Due to, for $\left.\forall n x-y N, A\right)_{\mathrm{n}}(I \text { and } a x N, A)_{\mathrm{n}}(I$ then $\mathrm{x}-\mathrm{y} \cap(N$, $A)_{\mathrm{n}}(I)$ and $a x \cap(N, A)_{\mathrm{n}}(I)$. Therefore $\cap(N, A)_{\mathrm{n}}(\mathrm{I})$ is a strong soft- $N$ submodule of $(\mathrm{H}, \mathrm{A})(\mathrm{I})$.

Remark 1. Let $(H, A)(I)$ be a strong soft-N G-mod on a soft-N ring $(G, A)(I)$ and let $(N, A)_{1}(I)$ and $(N, A)_{2}(I)$ be two distinct strong soft-N submodule of $(H, A)(I)$. Usually, $(N, A)_{1}(I) \cup(N, A)_{2}(I)$ is not a strong soft-N submodule of $(H, A)(I)$. On the other hand, in that case $(N, A)_{1}(I) \subseteq(N, A)_{2}(I)$ or $(N, A)_{2}(I) \supseteq(N, A)_{1}(I),(N$, $A)_{1}(I) U(N, A)_{2}(I)$ is a strong soft-N submodule of $(H, A)(I)$.

Definition 14. Let $(H, A)(I)$ and $(N, A)(I)$ be strong soft-N G-mod on a soft-N ring $(G, A)(I)$ and let $\sigma$ : $(H$, A) $(I) \rightarrow(N, A)(I)$ be a mapping of $(H, A)(I)$ into $(N, A)(I)$. $\sigma$ is called a soft-N G-mod homomorphism but only if the following conditions hold:

(i) $\sigma$ is a soft G-mod homomorphism.

(ii) $\sigma(I)=I$.

If $\sigma$ is a bijective soft-N G-mod homomorphism, $\sigma$ is called a soft-N G-mod isomorphism and it is shown as $(H, A)(I) \cong(N, A)(I)$.

Definition 15. Let $(H, A)(I)$ and $(N, A)(I)$ be strong soft-N G-mod on a soft-N ring $(G, A)(I)$ and let $\sigma$ : $(H$, A) $(I) \rightarrow(N, A)(I)$ be a soft-N G-mod homomorphism.

(i) The set $\{n(N, A)(I): \sigma(h)=n$ for some $h H, A)(I)\}$ is called the image of $\sigma$ and it is denoted by Imo.

(ii) The set $\left\{a_{m}(H, A)(I): \sigma\left(a_{m}\right)=0\right.$ for some $\left.a_{m} H(I)\right\}$ is called the kernel of $\sigma$ and it is denoted by Ker $\sigma$.

Example 5. Let $(H, A)(I)$ be a strong soft-N G-mod over a soft-N ring $(G, A)(I)$. 
(i) The mapping $\sigma:(H, A)(I) \rightarrow(H, A)$ (I) is described as $\sigma(a)=a \forall a \in(H, A)$ (I) is soft-N G-mod homomorphism. Furthermore Ker $\sigma=0$.

(ii) The mapping $\sigma:(H, A)(I) \rightarrow(H, A)(I)$ is described as $\sigma(a)=0 \forall a \in(H, A)$ (I) is soft-N G-mod homomorphism since $I \in(H, A)(I)$. On the other hand $\sigma(I) \neq 0$.

Theorem 8. Let $(H, A)(I)$ and $(N, A)(I)$ be strong soft-N G-mod on a soft-N ring $(G, A)(I)$. If $\sigma:(H, A)(I) \rightarrow$ $(N, A)(I)$ be a soft-N G-mod homomorphism, then

(i) The kernel of $\sigma$ is not a strong soft-N submodule of $(H, A)(I)$, on the other hand it is a soft submodule of $(H, A)$.

(ii) The image of $\sigma$ is a strong soft-N submodule of $(N, A)(I)$.

Proof. (i) Clearly, $I(\mathrm{H}, \mathrm{A})(\mathrm{I})$ and $\sigma(I) 0$. This implies that kernel of $\sigma$ does not contain $I$. Therefore Ker $\sigma$ is not a strong soft- $N$ submodule of $(\mathrm{H}, \mathrm{A})(\mathrm{I})$. But the kernel of $\sigma$ is a soft submodule of $(H, A)$ [26]. (ii) Open.

Theorem 9. Let $(N, A)(I)$ be a strong soft-N submodule of a strong soft-N G-mod $(M, A)(I)$ on a soft-N ring $(G, A)(I)$. If $\sigma:(H, A)(I) \rightarrow(H, A)(I) /(N, A)(I)$ is a mapping described as $\sigma(a)=a+(N, A)(I) \forall a \in(H, A)$ (I) then $\sigma$ is not a soft-N G-mod homomorphism.

Proof. We have $\sigma(I)=I+(N, A)(I)=(N, A)(I) \neq I$ from definition of $\sigma$. Therefore $\sigma$ is not a soft-N G-mod homomorphism.

Theorem 10. Let $(N, A)(I)$ be a strong soft-N submodule of a strong soft-N G-mod $(H, A)(I)$ on a soft-N ring $(G, A)(I)$ and let $\sigma:(H, A)(I) \rightarrow(K, A)(I)$ be a soft-N G-mod homomorphism on $(G, A)(I)$. If $\sigma_{(N, A)(I)}:(N, A)$ $(I) \rightarrow(K, A)(I)$ is the restriction of $\sigma$ to $(N, A)(I)$ is described as $\sigma_{(N, A)(I)}(n)=\sigma(n)$. Then:

(i) $\operatorname{Ker} \sigma \cap(N, A)(I)=(N, A)(I) \operatorname{Ker} \sigma_{(N, A)(I)}$

(ii) $\sigma(N, A)(I)$ is a soft-N G-mod homomorphism.

(iii) $\sigma((N, A)(I))=\operatorname{Im} \sigma_{(N, A)(I)}$.

Proof. (i) It is clear from the homological properties of $\sigma$.

(i) The restriction of $\sigma$ to $(N, A)(I), \sigma(N, A)(I)$ is a soft-N G-mod homomorphism since $\sigma$ is a soft- $N$ G-mod homomorphism.

(ii) It is obtain easily from definition of the $\sigma$.

Remark 2. If $(H, A)(I)$ and $(N, A)(I)$ are strong soft-N G-mod on a soft-N ring $(G, A)(I)$. Let $\sigma, \mu$ : (H, A) (I) $\rightarrow(N, A)(I)$ are soft-N G-mod homomorphisms. Then $(\sigma+\mu)$ and $(k \sigma)$ are not soft-N G-mod homomorphisms for any $k \in(G, A)(I)$. Because $(\sigma+\mu)(I)=\sigma(I)+\mu(I)=I+I=2 I \neq I$ and $(k \sigma)(I)=k \sigma(I)=k I \neq I$ for every $k$ $\in(G, A)(I)$.

Therefore, If Hom $((H, A)(I),(N, A)(I))$ is the collection of every soft-N G-mod homomorphisms from $(H, A)(I)$ into $(N, A)(I)$ and it is not a soft-N G-mod on $(G, A)(I)$. That is completely different from which is attainable in the classical G-mod.

Definition 16. Let $(N, A)(I),(H, A)(I)$ and $(K, A)(I)$ be strong soft-N G-mod on a soft-N ring $(G, A)(I)$. Let $\sigma:(K, A)(I) \rightarrow(H, A)(I)$ and $\mu:(H, A)(I) \rightarrow(N, A)(I)$ be soft-N G-mod homomorphism., the composition $\mu \sigma:$ $(K, A)(I) \rightarrow(N, A)(I)$ is described as $\mu(\sigma(k))=\mu \sigma(k)$ for $\forall k \in(K, A)(I)$.

Theorem 11. Let $(N, A)(I),(H, A)(I)$ and $(K, A)(I)$ be strong soft-N G-mod on a soft-N ring $(G, A)(I)$. If $\sigma$ : $(K, A)(I) \rightarrow(H, A)(I)$ and $\mu:(H, A)(I) \rightarrow(N, A)(I)$ are soft-N G-mod homomorphisms, then the composition $\sigma$ and $\mu, \mu \sigma:(K, A)(I) \rightarrow(N, A)(I)$ is a soft-N G-mod homomorphism.

Proof. Obviously, $\mu \sigma$ is a soft G-mod homomorphism for $k I K, A)(I)$ and we have:

$$
\begin{gathered}
\mu \sigma(I)=\mu(\sigma(I)) \\
=\mu(I) \\
=I .
\end{gathered}
$$


Therefore, $\mu \sigma$ is a soft-N G-mod homomorphism.

Corollary 1. Let $\mathrm{HOM}((H, A)(I),(H, A)(I))$ be the collection of every soft-N G-mod homomorphisms from ( $H$, A) (I) onto $(H, A)(I)$. Then we have $(\sigma \mu) \lambda=\sigma(\mu \lambda)$ for $\forall \lambda, \mu, \sigma, \in \operatorname{HOM}((H, A)(I),(H, A)(I))$.

Proof. It is open from the Theorem 11.

Theorem 12. Assume that $(K, A)(I),(H, A)(I)$ and $(N, A)(I)$ are strong soft-N G-mod on a soft-N ring $(G, A)$ $(I)$. and $\sigma:(K, A)(I) \rightarrow(H, A)(I)$ and $\mu:(H, A)(I) \rightarrow(N, A)(I)$ are soft-N G-mod homomorphisms. Then,

(i) If $\mu \sigma$ is surjective, then $\sigma$ is surjective.

(ii) If $\mu \sigma$ is injective, then $\mu$ is injective.

(iii) If $\mu$ and $\sigma$ are injective, then $\mu \sigma$ is injective.

Proof. It is clear from the properties of mappings.

\section{Conclusions}

This paper is mainly focused on soft neutrosophic module. Firstly, we have examined the generalization and some structural features of soft- $N$ modules in the appropriate examples. Then we described and exemplified weak soft- $N$ modules and strong soft- $N$ modules. We proved that each strong soft -N G-mod is a weak soft-N G-mod and each weak (strong) soft-N G-mod is a soft G-mod. Finally, we described the soft- $N$ module homomorphism and the soft- $N$ module isomorphism and examined some properties of the soft- $N$ module homomorphism. Using the results obtained, researchers can investigate the homological characteristics of these modules.

Author Contributions: Investigation, M.B.; Writing— review \& editing, N.O.

Conflicts of Interest: The authors declare no conflict of interest.

\section{References}

1. Molodtsov, D. Soft Set Theory-First result. Comput. Math. Appl. 1999, 37, 19-31. [CrossRef]

2. Shabir, M.; Ali, M.; Naz, M.; Smarandache, F. Soft Neutrosophic Group. Neutrosophic Sets Syst. 2013, 1, 13-25.

3. Ali, M.; Smarandache, F.; Shabir, M.; Naz, M. Soft Neutrosophic Ring and Soft Neutrosophic Field. Neutrosophic Sets Syst. 2014, 3, 53-57.

4. Ali, M.; Smarandache, F.; Vladareanu, L.; Shabir, M. Generalization of Soft Neutrosophic Rings and Soft Neutrosophic Fields. Neutrosophic Sets Syst. 2014, 6, 35-41.

5. Agboola, A.A.A.; Akinola, A.D.; Oyebola, O.Y. Neutrosophic Rings I. Int. J. Math. Comb. 2011, 4, 1-14.

6. Yetkin, E.; Olgun, N. A New Type Fuzzy Module over Fuzzy Rings. Sci. World J. 2014, $2014,730932$. [CrossRef] [PubMed]

7. Sharp, R.Y. Steps in Commutative Algebra, 2nd ed.; Cambridge University Press: Cambridge, UK, 2000.

8. Bera, T.; Mahapatra, N.K. On Neutrosophic Soft Linear Spaces. Fuzzy Inf. Eng. 2017, 9, 299-324. [CrossRef]

9. Acar, U.; Koyuncu, F.; Tanay, B. Soft Sets and Soft Ring. Comput. Math. Appl. 2010, 59, 3458-3463. [CrossRef]

10. Uluçay, V.; Sahin, M.; Olgun, N.; Oztekin, O.; Emniyet, A. Generalized Fuzzy $\sigma$-Algebra and Generalized Fuzzy Measure on Soft Sets. Indian J. Sci. Technol. 2016, 9, 1-7. [CrossRef]

11. Uluçay, V.; Şahin, M.; Olgun, N. Soft Normed Rings. SpringerPlus 2016, 5, 1950-1956. [CrossRef]

12. Vasantha Kandasamy, W.B.; Smarandache, F. Neutrosophic Rings; Hexis: Phoenix, AZ, USA, 2006.

13. Uluçay, V.; Şahin, M.; Olgun, N. Normed Z-Modules. Int. J. Pure Appl. Math. 2017, 112, 425-435.

14. Şahin, M.; Olgun, N.; Uluçay, V. Normed Quotient Rings. New Trends Math. Sci. 2018, 6, 52-58.

15. Şahin, M.; Olgun, N.; Kargın, A.; Uluçay, V. Isomorphism theorems for soft G-modules. Afr. Mat. 2018, 29, 1-8. [CrossRef]

16. Smarandache, F.; Şahin, M.; Kargın, A. Neutrosophic Triplet G-Module. Mathematics 2018, 6, 53. [CrossRef]

17. Şahin, M.; Kargın, A. Neutrosophic Triplet Inner Product. Neutrosophic Oper. Res. 2017, 2, $193-205$.

18. Şahin, M.; Uluçay, V.; Olgun, N.; Kilicman, A. On Neutrosophic Soft Lattices. Afr. Mat. 2017, 28, 379-388.

19. Sahin, M.; Deli, I.; Ulucay, V. Similarity measure of bipolar neutrosophic sets and their application to multiple criteria decision making. Neural Comput. Appl. 2016, 29, 739-748. 
20. Hassan, N.; Ulucay, V.; Sahin, M. Q-Neutrosophic Soft Expert Set and Its Application in Decision Making. Int. J. Fuzzy Syst. Appl. 2018, 7, 37-61. [CrossRef]

21. Uluçay, V.; Şahin, M.; Hassan, N. Generalized Neutrosophic Soft Expert Set for Multiple-Criteria Decision-Making. Symmetry 2018, 10, 437. [CrossRef]

22. Agboola, A.A.A.; Akinleye, S.A. Neutrosophic Vector Space. Neutrosophic Sets Syst. 2014, 4, 9-18.

23. Aktas, H.; Çağman, N. Soft Sets and Soft Groups. Inf. Sci. 2007, 177, 2726-2735. [CrossRef]

24. Olgun, N.; Bal, M. Neutrosophic Modules. Neutrosophic Operational Research Volume II; Pons Publishing House: Bruxelles, Belgium, 2016; pp. 181-192.

25. Maji, P.K.; Biswas, R.; Roy, A.R. Soft set theory. Comput. Math. Appl. 2003, 45, 555-562. [CrossRef]

26. Sun, Q.-M.; Zhang, Z.-L.; Liu, J. Soft sets and soft modules. Lect. Notes Comput. Sci. 2008, 5009, 403-409.

(C) 2018 by the authors. Licensee MDPI, Basel, Switzerland. This article is an open access article distributed under the terms and conditions of the Creative Commons Attribution (CC BY) license (http:/ / creativecommons.org/licenses/by/4.0/). 